
where, $2 \pi r$ is the circumference of the Equilibrium Orbit (E.O.), $s=r \theta$ is the distance along the E.O. measured from a fixed reference point,

$$
k(s)=\frac{e}{c P}\left(\frac{\partial B_{y}}{\partial x}\right)
$$

is proportional to the quadrupole strength on the E.O. $(x=0, y=0)$ and inversely proportional to the momentum, $P$,

$$
\begin{array}{cc}
\psi_{x}=2 \mu_{x}+\left(p-2 Q_{x}\right) \theta, & \psi_{y}=2 \mu_{y}+\left(p-2 Q_{y}\right) \theta \\
\mu_{x}(s)=\int_{0}^{s} \frac{d s^{\prime}}{\beta_{x}\left(s^{\prime}\right)}, & \mu_{y}(s)=\int_{0}^{s} \frac{d s^{\prime}}{\beta_{y}\left(s^{\prime}\right)}
\end{array}
$$

are the betatron phase advances,

$$
C=\frac{1}{8 \pi r}
$$

and $Q_{x}$ and $Q_{y}$ are the unperturbed horizontal and vertical tunes. These resonances can produce unlimited growth in the amplitudes of the betatron oscillations whenever the tunes are such that

$$
-\frac{W}{2}<2 Q_{x, y}-p<\frac{W}{2}
$$

where,

$$
W=4 r\left|\kappa_{x, y}\right|
$$

is the stopband width.

\subsection{Resonances $3 Q_{x}=p$ and $Q_{x}+2 Q_{y}=p$}

For the $3 Q_{x}=p$ and $Q_{x}+2 Q_{y}=p$ resonances the excitation coefficients are respectively

$$
\kappa_{x}=C \int_{0}^{2 \pi r} k(s) \beta_{x}^{3 / 2} e^{i \psi_{x}} d s, \quad \kappa_{x y}=-3 C \int_{0}^{2 \pi r} k(s) \beta_{x}^{1 / 2} \beta_{y} e^{i \psi_{x y}} d s
$$

where,

$$
k(s)=\frac{e}{c P}\left(\frac{\partial^{2} B_{y}}{\partial x^{2}}\right)
$$

is proportional to the sextupole strength on the E.O.,

$$
\psi_{x}=3 \mu_{x}+\left(p-3 Q_{x}\right) \theta, \quad \psi_{x y}=\mu_{x}+2 \mu_{y}+\left(p-Q_{x}-2 Q_{y}\right) \theta
$$




$$
C=\frac{1}{2 \pi r} \frac{1}{2 \sqrt{2}} \frac{1}{6}
$$

The stopband widths for these resonances are

$$
\begin{gathered}
W_{x}=\frac{18}{\sqrt{2 \pi}} r\left|\kappa_{x}\right| \sqrt{\epsilon_{x}}, \\
W_{x y}=\sqrt{\frac{2}{\pi}} r\left|\kappa_{x y}\right| \frac{1}{\sqrt{\epsilon_{x}}}\left(\epsilon_{y}+4 \epsilon_{x}\right),
\end{gathered}
$$

where $\epsilon_{\mathfrak{x}}, \epsilon_{y}$ are the initial emittances.

\subsection{Resonances $3 Q_{y}=p$ and $2 Q_{x}+Q_{y}=p$}

The excitation coefficients and stopband widths for the $3 Q_{y}=p$ and $2 Q_{x}+Q_{y}=p$ resonances may be obtained from equations (8), (10), and (12-13) by interchanging $x$ and $y$ and replacing $k(s)$ with

$$
k(s)=\frac{e}{c P}\left(\frac{\partial^{2} B_{x}}{\partial x^{2}}\right)
$$

which is proportional to the skew sextupole strength on the E.O.

\subsection{Comments}

Careful inspection of the equations for the excitation coefficients, $\kappa$, shows that each $\kappa$ is essentially proportional to the $p$ th harmonic in the azimuthal variation of $k(s)$ around the machine. The real and imaginary parts of $\kappa$ are then the $\cos$ and sin components of this harmonic. The resonances discussed in sections 2.1-2.3 are therefore excited by the $p$ th harmonic in the azimuthal variations of the quadrupole, sextupole, and skew sextupole fields around the machine.

Gaussian units (cm, gram, second, erg, gauss, statcoulomb) are employed in the equations for $k(s)$ given in sections 2.1-2.3. Thus if the momentum, $P$, is expressed in $\mathrm{eV} / \mathrm{c}$ then $c P / e=3335.641$ gauss-cm per $\mathrm{MeV}$, or $c P / e=3.335641$ tesla-m per $\mathrm{GeV}$. 


\section{Correction Schemes}

Any naturally occuring fields in the machine, or fields due to imperfections, which produce nonzero values of the excitation coefficients, $\kappa$, can excite resonances resulting in beam loss. We call each $\kappa$ produced by these fields an intrinsic excitation coefficient of the machine and denote it by $\kappa_{0}$. To cancel each $\kappa_{0}$, so that the resonances can not be excited, correction elements located at various positions, $s_{j}$, in the ring are excited with currents, $I_{j}$, in such a way that they produce a $\kappa$ equal to $-\kappa_{0}$. When this is done we say that the resonances have been corrected.

\subsection{Correction of resonances $2 Q_{x}=p$ and $2 Q_{y}=p$}

Suppose there are $N$ identical correction quadrupoles located at positions, $s_{j}$, and excited with currents $I_{j}$. If the integrated strength of each quadrupole is $Q$ gauss/amp, then the set of quadrupoles will produce excitation coefficients

$$
\kappa_{x}=C\left(\frac{e Q}{c P}\right) \sum_{j=1}^{N} \beta_{x j} I_{j} e^{i \psi_{x j}}, \quad \kappa_{y}=-C\left(\frac{e Q}{c P}\right) \sum_{j=1}^{N} \beta_{y j} I_{j} e^{i \psi_{y j}}
$$

in which

$$
\begin{aligned}
\beta_{x j}=\beta_{x}\left(s_{j}\right), & \beta_{y j}=\beta_{y}\left(s_{j}\right) \\
\psi_{x j}=2 \mu_{x}\left(s_{j}\right)+\left(p-2 Q_{x}\right) \theta_{j}, & \psi_{y j}=2 \mu_{y}\left(s_{j}\right)+\left(p-2 Q_{y}\right) \theta_{j},
\end{aligned}
$$

and equations (1-4) have been employed in the thin lens approximation. Generally it is necessary to correct both resonances simultaneously since some particles in the beam may be near the $2 Q_{x}=p$ resonance while others are near the $2 Q_{y}=p$ resonance. This is especially true near injection where the beam is spread over a large region of tune space due to space charge detuning. In general, then, the positions, $s_{j}$, of the quadrupoles must be chosen so that it is always possible to find a set of currents, $I_{j}$, which produce the values of $\kappa_{x}$ and $\kappa_{y}$ required to correct both resonances at the same time. Since each $\kappa$ has a real and an imaginary part, we see from equations (15) that four correction elements are needed.

\subsubsection{Correction of one resonance only}

Before considering the general case let us consider the special case in which it is necessary to correct only one of the resonances, say $2 Q_{x}=p$. Then 
only two correction elements are needed, and taking $N=2$ the first of equations (15) becomes

$$
\left(\begin{array}{c}
C X \\
S X
\end{array}\right)=C\left(\frac{e Q}{c P}\right)\left(\begin{array}{cc}
C_{1} & C_{2} \\
S_{1} & S_{2}
\end{array}\right)\left(\begin{array}{c}
\beta_{x 1} I_{1} \\
\beta_{x 2} I_{2}
\end{array}\right)
$$

where $C X$ and $S X$ are respectively the cos (real) and $\sin$ (imaginary) parts of $\kappa_{x}, C_{j}=\cos \left(\psi_{x j}\right)$, and $S_{j}=\sin \left(\psi_{x j}\right)$. Solving for $I_{1}$ and $I_{2}$ we find

$$
\left(\begin{array}{c}
\beta_{x 1} I_{1} \\
\beta_{x 2} I_{2}
\end{array}\right)=\frac{1}{C}\left(\frac{c P}{e Q}\right) \frac{1}{S_{12}}\left(\begin{array}{rr}
S_{2} & -C_{2} \\
-S_{1} & C_{1}
\end{array}\right)\left(\begin{array}{c}
C X \\
S X
\end{array}\right)
$$

where $S_{12}=C_{1} S_{2}-S_{1} C_{2}=\sin \left(\psi_{x 2}-\psi_{x 1}\right)$. Here we see that the amount of current required to produce a given $\kappa_{x}$ is proportional to $1 / S_{12}$ which becomes infinite whenever the phase difference, $\psi_{x 2}-\psi_{x 1}$, is an integral multiple of $\pi$. If we define

$$
\phi_{x j}=\phi_{x}\left(s_{j}\right)=\mu_{x}\left(s_{j}\right) / Q_{x}, \quad \phi_{y j}=\phi_{y}\left(s_{j}\right)=\mu_{y}\left(s_{j}\right) / Q_{y}
$$

then near the $2 Q_{\mathfrak{x}}=p$ resonance the phase difference

$$
\psi_{x 2}-\psi_{x 1}=p \phi_{x}\left(s_{2}\right)-p \phi_{x}\left(s_{1}\right)=p\left(\phi_{x 2}-\phi_{x 1}\right) .
$$

Thus the effectiveness of the currents, $I_{1}$ and $I_{2}$, in producing the desired corrections is proportional to $\left|S_{12}\right|=\left|\sin \left(p \phi_{x 2}-p \phi_{x 1}\right)\right|$ and we see that one must avoid positions for which $p\left(\phi_{x 2}-\phi_{x 1}\right)$ is an integral multiple of $\pi$. The optimum positions-i.e. those for which the least amount of current is required to produce the desired corrections-are those for which $p\left(\phi_{x 2}-\phi_{x 1}\right)$ is an odd multiple of $\pi / 2$, and $\beta_{x 1}$ and $\beta_{x 2}$ are beta maximums.

\subsubsection{Correction of both resonances simultaneously}

Let us now return to the general problem of correcting both resonances simultaneously. In this case four correction elements are needed, and with $N=4$ equations (15) become

$$
\left(\begin{array}{c}
C X \\
S X \\
C Y \\
S Y
\end{array}\right)=C\left(\frac{e Q}{c P}\right) \mathrm{M}\left(\begin{array}{c}
I_{1} \\
I_{2} \\
I_{3} \\
I_{4}
\end{array}\right)
$$


where,

$$
\mathbf{M}=\left(\begin{array}{rrrr}
\beta_{x 1} C_{x 1} & \beta_{x 2} C_{x 2} & \beta_{x 3} C_{x 3} & \beta_{x 4} C_{x 4} \\
\beta_{x 1} S_{x 1} & \beta_{x 2} S_{x 2} & \beta_{x 3} S_{x 3} & \beta_{x 4} S_{x 4} \\
-\beta_{y 1} C_{y 1} & -\beta_{y 2} C_{y 2} & -\beta_{y 3} C_{y 3} & -\beta_{y 4} C_{y 4} \\
-\beta_{y 1} S_{y 1} & -\beta_{y 2} S_{y 2} & -\beta_{y 3} S_{y 3} & -\beta_{y 4} S_{y 4}
\end{array}\right),
$$

$C X$ and $S X$ are the cos and sin parts of $\kappa_{x}, C Y$ and $S Y$ are the cos and $\sin$ parts of $\kappa_{y}, C_{x j}=\cos \left(\psi_{x j}\right), S_{x j}=\sin \left(\psi_{x j}\right), C_{y j}=\cos \left(\psi_{y j}\right)$, and $S_{y j}=\sin \left(\psi_{y j}\right)$. The currents which produce the desired corrections are then

$$
\left(\begin{array}{c}
I_{1} \\
I_{2} \\
I_{3} \\
I_{4}
\end{array}\right)=\frac{1}{C}\left(\frac{c P}{e Q}\right) \mathbf{M}^{-1}\left(\begin{array}{c}
C X \\
S X \\
C Y \\
S Y
\end{array}\right)
$$

Here we see that the effectiveness of the correctors in producing the desired corrections depends on the inverse of the matrix $M$ which is, in general, rather complicated. However, if one makes some assumptions about the machine lattice and the placement of the correctors, then both $\mathbf{M}$ and its inverse become much simpler. The determination of the effectiveness of the correctors then becomes rather straight forward. The conditions under which the following simplifing assumptions are valid will be discussed in section 3.4 .

In the previous section we found that the $2 Q_{x}=p$ resonance is most effectively corrected when the two correction elements are located at horizontal beta maximums. Thus, if we wish also to correct the $2 Q_{y}=p$ resonance, two correction elements should be placed at vertical beta maximums. We therefore take positions $s_{1}$ and $s_{2}$ to be vertical beta maximums and positions $s_{3}$ and $s_{4}$ to be horizontal beta maximums. We shall also take $s_{1}=0$ and assume that

$$
\phi_{x}\left(s_{j}\right)=\phi_{y}\left(s_{j}\right)=\phi\left(s_{j}\right)=\phi_{j}
$$

where $\phi_{x}\left(s_{j}\right)$ and $\phi_{y}\left(s_{j}\right)$ are the normalized betatron phase advances defined in equations (18). Then near the resonances we have

$$
\psi_{x j}=\psi_{y j}=p \phi_{j}
$$

and therefore

$$
C_{x j}=C_{y j}=C_{j}=\cos \left(p \phi_{j}\right), \quad S_{x j}=S_{y j}=S_{j}=\sin \left(p \phi_{j}\right) .
$$


We shall also assume that

$$
\begin{aligned}
& \beta_{x 1}=\beta_{x 2}=a, \quad \beta_{x 3}=\beta_{x 4}=A, \\
& \beta_{y 1}=\beta_{y 2}=B, \quad \beta_{y 3}=\beta_{y 4}=b,
\end{aligned}
$$

where $(a, b)$ and $(A, B)$ are respectively values of beta minima and beta maxima in the machine lattice. With these assumptions the matrix, $\mathbf{M}$, becomes

$$
\mathbf{M}=\left(\begin{array}{rrrr}
a C_{1} & a C_{2} & A C_{3} & A C_{4} \\
a S_{1} & a S_{2} & A S_{3} & A S_{4} \\
-B C_{1} & -B C_{2} & -b C_{3} & -b C_{4} \\
-B S_{1} & -B S_{2} & -b S_{3} & -b S_{4}
\end{array}\right)
$$

Introducing

$$
\mathbf{m}=\left(\begin{array}{cc}
C_{1} & C_{2} \\
S_{1} & S_{2}
\end{array}\right), \quad \mathbf{n}=\left(\begin{array}{cc}
C_{3} & C_{4} \\
S_{3} & S_{4}
\end{array}\right)
$$

we then have

$$
\mathbf{M}=\left(\begin{array}{rr}
a \mathbf{m} & A \mathbf{n} \\
-B \mathbf{m} & -b \mathbf{n}
\end{array}\right)=\left(\begin{array}{rr}
a & A \\
-B & -b
\end{array}\right)\left(\begin{array}{cc}
\mathbf{m} & \mathbf{0} \\
\mathbf{0} & \mathbf{n}
\end{array}\right)
$$

and

$$
\mathbf{M}^{-1}=\frac{1}{A B-a b}\left(\begin{array}{ll}
\mathbf{m}^{-1} & 0 \\
\mathbf{0} & \mathbf{n}^{-1}
\end{array}\right)\left(\begin{array}{rr}
-b & -A \\
B & a
\end{array}\right)
$$

where

$$
\begin{gathered}
\mathbf{m}^{-1}=\frac{1}{S_{12}}\left(\begin{array}{rr}
S_{2} & -C_{2} \\
-S_{1} & C_{1}
\end{array}\right), \quad \mathbf{n}^{-1}=\frac{1}{S_{34}}\left(\begin{array}{rr}
S_{4} & -C_{4} \\
-S_{3} & C_{3}
\end{array}\right) \\
S_{12}=C_{1} S_{2}-S_{1} C_{2}=\sin \left(p \phi_{2}-p \phi_{1}\right) \\
S_{34}=C_{3} S_{4}-S_{3} C_{4}=\sin \left(p \phi_{4}-p \phi_{3}\right) .
\end{gathered}
$$

Putting (24) into (21) we obtain

$$
\begin{gathered}
\left(\begin{array}{c}
I_{1} \\
I_{2}
\end{array}\right)=\frac{-g}{(A B-a b) S_{12}}\left(\begin{array}{rr}
S_{2} & -C_{2} \\
-S_{1} & C_{1}
\end{array}\right)\left(\begin{array}{c}
b C X+A C Y \\
b S X+A S Y
\end{array}\right), \\
\left(\begin{array}{c}
I_{3} \\
I_{4}
\end{array}\right)=\frac{g}{(A B-a b) S_{34}}\left(\begin{array}{rr}
S_{4} & -C_{4} \\
-S_{3} & C_{3}
\end{array}\right)\left(\begin{array}{c}
B C X+a C Y \\
B S X+a S Y
\end{array}\right)
\end{gathered}
$$


where

$$
g=\frac{1}{C}\left(\frac{c P}{e Q}\right)
$$

Here we see that the effectiveness of currents $I_{1}$ and $I_{2}$ in producing the desired corrections is proportional to $\left|S_{12}\right|=\left|\sin \left(p \phi_{2}-p \phi_{1}\right)\right|$. Likewise the effectiveness of currents $I_{3}$ and $I_{4}$ is proportional to $\left|S_{34}\right|=\left|\sin \left(p \phi_{4}-p \phi_{3}\right)\right|$. Thus, corrector positions for which either $p\left(\phi_{2}-\phi_{1}\right)$ or $p\left(\phi_{4}-\phi_{3}\right)$ is an integral multiple of $\pi$ must be avoided. The optimum positions are those for which $p\left(\phi_{2}-\phi_{1}\right)$ and $p\left(\phi_{4}-\phi_{3}\right)$ are odd multiples of $\pi / 2$. We also see that the effectiveness of the currents is proportional to $A B-a b$, which is zero when $A B=a b$. This is consistent with our earlier assumption that two correction elements should be placed at horizontal beta maximums and two at vertical beta maximums.

\subsection{Correction of resonances $3 Q_{x}=p$ and $Q_{x}+2 Q_{y}=p$}

As in section 3.1 we suppose that there are $N$ identical correction elements-sextupoles in this case-located at positions, $s_{j}$, and excited with currents $I_{j}$. If the integrated strength of each sextupole is $S$ gauss $/ \mathrm{cm}$ per amp, then the set of sextupoles will produce excitation coefficients

$$
\kappa_{x}=C\left(\frac{e S}{c P}\right) \sum_{j=1}^{N} \beta_{x j}^{3 / 2} I_{j} e^{i \psi_{x j}}, \quad \frac{1}{3} \kappa_{x y}=-C\left(\frac{e S}{c P}\right) \sum_{j=1}^{N} \beta_{x j}^{1 / 2} \beta_{y j} I_{j} e^{i \psi_{y j}}
$$

in which

$$
\begin{gathered}
\beta_{x j}=\beta_{x}\left(s_{j}\right), \quad \beta_{y j}=\beta_{y}\left(s_{j}\right), \\
\psi_{x j}=3 \mu_{x}\left(s_{j}\right)+\left(p-3 Q_{x}\right) \theta_{j}, \\
\psi_{y j}=\mu_{x}\left(s_{j}\right)+2 \mu_{y}\left(s_{j}\right)+\left(p-Q_{x}-2 Q_{y}\right) \theta_{j},
\end{gathered}
$$

and equations (8-11) have been employed in the thin lens approximation. As with the half-integer resonances it is generally necessary to correct the $3 Q_{x}=p$ and $Q_{x}+2 Q_{y}=p$ resonances simultaneously. Since each $\kappa$ has a real and an imaginary part we see from equations (26) that four correction elements are needed. Taking $N=4$ equations (26) become

$$
\left(\begin{array}{c}
C X \\
S X \\
C Y \\
S Y
\end{array}\right)=C\left(\frac{e S}{c P}\right) \mathrm{M}\left(\begin{array}{c}
I_{1} \\
I_{2} \\
I_{3} \\
I_{4}
\end{array}\right)
$$


where

$$
\mathbf{M}=\left(\begin{array}{rrrr}
\beta_{x 1}^{3 / 2} C_{x 1} & \beta_{x 2}^{3 / 2} C_{x 2} & \beta_{x 3}^{3 / 2} C_{x 3} & \beta_{x 4}^{3 / 2} C_{x 4} \\
\beta_{x 1}^{3 / 2} S_{x 1} & \beta_{x 2}^{3 / 2} S_{x 2} & \beta_{x 3}^{3 / 2} S_{x 3} & \beta_{x 4}^{3 / 2} S_{x 4} \\
-\beta_{x 1}^{1 / 2} \beta_{y 1} C_{y 1} & -\beta_{x 2}^{1 / 2} \beta_{y 2} C_{y 2} & -\beta_{x 3}^{1 / 2} \beta_{y 3} C_{y 3} & -\beta_{x 4}^{1 / 2} \beta_{y 4} C_{y 4} \\
-\beta_{x 1}^{1 / 2} \beta_{y 1} S_{y 1} & -\beta_{x 2}^{1 / 2} \beta_{y 2} S_{y 2} & -\beta_{x 3}^{1 / 2} \beta_{y 3} S_{y 3} & -\beta_{x 4}^{1 / 2} \beta_{y 4} S_{y 4}
\end{array}\right)
$$

$C X$ and $S X$ are the $\cos$ and $\sin$ parts of $\kappa_{x}, C Y$ and $S Y$ are the cos and $\sin$ parts of $\kappa_{x y} / 3, C_{x j}=\cos \left(\psi_{x j}\right), S_{x j}=\sin \left(\psi_{x j}\right), C_{y j}=\cos \left(\psi_{y j}\right)$, and $S_{y j}=\sin \left(\psi_{y j}\right)$. The currents which produce the desired corrections are then

$$
\left(\begin{array}{c}
I_{1} \\
I_{2} \\
I_{3} \\
I_{4}
\end{array}\right)=\frac{1}{C}\left(\frac{c P}{e S}\right) \mathbf{M}^{-1}\left(\begin{array}{c}
C X \\
S X \\
C Y \\
S Y
\end{array}\right)
$$

We now make some assumptions, as before, which simplify the form of $M$ and make the determination of the effectiveness of a given set of correction elements straight forward. The conditions under which these assumptions are valid will be discussed in section 3.4. As in section 3.1 .2 we take positions $s_{1}$ and $s_{2}$ to be vertical beta maximums and positions $s_{3}$ and $s_{4}$ to be horizontal beta maximums. We also take $s_{1}=0$ and assume that

$$
\phi_{x}\left(s_{j}\right)=\phi_{y}\left(s_{j}\right)=\phi\left(s_{j}\right)=\phi_{j}
$$

where $\phi_{x}\left(s_{j}\right)$ and $\phi_{y}\left(s_{j}\right)$ are the normalized betatron phase advances defined in equations (18). Then near the resonances we have

$$
\psi_{x j}=\psi_{y j}=p \phi_{j}
$$

and therefore

$$
C_{x j}=C_{y j}=C_{j}=\cos \left(p \phi_{j}\right), \quad S_{x j}=S_{y j}=S_{j}=\sin \left(p \phi_{j}\right)
$$

We also assume that

$$
\begin{aligned}
& \beta_{x 1}=\beta_{x 2}=a, \quad \beta_{x 3}=\beta_{x 4}=A \\
& \beta_{y 1}=\beta_{y 2}=B,
\end{aligned}
$$


where $(a, b)$ and $(A, B)$ are respectively values of beta minima and beta maxima in the machine lattice. With these assumptions the matrix, $\mathbf{M}$, becomes

$$
\mathbf{M}=\left(\begin{array}{rrrr}
a^{3 / 2} C_{1} & a^{3 / 2} C_{2} & A^{3 / 2} C_{3} & A^{3 / 2} C_{4} \\
a^{3 / 2} S_{1} & a^{3 / 2} S_{2} & A^{3 / 2} S_{3} & A^{3 / 2} S_{4} \\
-a^{1 / 2} B C_{1} & -a^{1 / 2} B C_{2} & -A^{1 / 2} b C_{3} & -A^{1 / 2} b C_{4} \\
-a^{1 / 2} B S_{1} & -a^{1 / 2} B S_{2} & -A^{1 / 2} b S_{3} & -A^{1 / 2} b S_{4}
\end{array}\right)
$$

Introducing

$$
\mathbf{m}=\left(\begin{array}{cc}
C_{1} & C_{2} \\
S_{1} & S_{2}
\end{array}\right), \quad \mathbf{n}=\left(\begin{array}{cc}
C_{3} & C_{4} \\
S_{3} & S_{4}
\end{array}\right)
$$

we then have

$$
\mathbf{M}=\left(\begin{array}{rr}
a^{3 / 2} & A^{3 / 2} \\
-a^{1 / 2} B & -A^{1 / 2} b
\end{array}\right)\left(\begin{array}{cc}
\mathbf{m} & 0 \\
\mathbf{0} & \mathbf{n}
\end{array}\right)
$$

and

$$
\mathbf{M}^{-1}=\frac{1}{a^{1 / 2} A^{1 / 2}(A B-a b)}\left(\begin{array}{ll}
\mathbf{m}^{-1} & \mathbf{0} \\
\mathbf{0} & \mathbf{n}^{-1}
\end{array}\right)\left(\begin{array}{rr}
-A^{1 / 2} b & -A^{3 / 2} \\
a^{1 / 2} B & a^{3 / 2}
\end{array}\right)
$$

where

$$
\begin{gathered}
\mathbf{m}^{-1}=\frac{1}{S_{12}}\left(\begin{array}{rr}
S_{2} & -C_{2} \\
-S_{1} & C_{1}
\end{array}\right), \quad \mathbf{n}^{-1}=\frac{1}{S_{34}}\left(\begin{array}{rr}
S_{4} & -C_{4} \\
-S_{3} & C_{3}
\end{array}\right) \\
S_{12}=C_{1} S_{2}-S_{1} C_{2}=\sin \left(p \phi_{2}-p \phi_{1}\right), \\
S_{34}=C_{3} S_{4}-S_{3} C_{4}=\sin \left(p \phi_{4}-p \phi_{3}\right) .
\end{gathered}
$$

Putting (32) into (29) we obtain

$$
\begin{gathered}
\left(\begin{array}{c}
I_{1} \\
I_{2}
\end{array}\right)=\frac{-g}{a^{1 / 2}(A B-a b) S_{12}}\left(\begin{array}{rr}
S_{2} & -C_{2} \\
-S_{1} & C_{1}
\end{array}\right)\left(\begin{array}{c}
b C X+A C Y \\
b S X+A S Y
\end{array}\right), \\
\left(\begin{array}{c}
I_{3} \\
I_{4}
\end{array}\right)=\frac{g}{A^{1 / 2}(A B-a b) S_{34}}\left(\begin{array}{rr}
S_{4} & -C_{4} \\
-S_{3} & C_{3}
\end{array}\right)\left(\begin{array}{c}
B C X+a C Y \\
B S X+a S Y
\end{array}\right),
\end{gathered}
$$

where

$$
g=\frac{1}{C}\left(\frac{c P}{e S}\right) .
$$


Here we see, as before, that the effectiveness of currents $I_{1}$ and $I_{2}$ in producing the desired corrections is proportional to $\left|S_{12}\right|=\left|\sin \left(p \phi_{2}-p \phi_{1}\right)\right|$. Likewise the effectiveness of currents $I_{3}$ and $I_{4}$ is proportional to $\left|S_{34}\right|=\left|\sin \left(p \phi_{4}-p \phi_{3}\right)\right|$. Corrector positions for which either $p\left(\phi_{2}-\phi_{1}\right)$ or $p\left(\phi_{4}-\phi_{3}\right)$ is an integral multiple of $\pi$ must therefore be avoided. The optimum positions are those for which $p\left(\phi_{2}-\phi_{1}\right)$ and $p\left(\phi_{4}-\phi_{3}\right)$ are odd multiples of $\pi / 2$. We also see that the effectiveness of the currents is proportional to $A B-a b$, which is zero when $A B=a b$. This is consistent with our assumption that two correction elements should be placed at horizontal beta maximums and two at vertical beta maximums.

\subsection{Correction of resonances $3 Q_{y}=p$ and $2 Q_{x}+Q_{y}=p$}

The formulae for the correction of the $3 Q_{y}=p$ and $2 Q_{x}+Q_{y}=p$ resonances may be obtained from the formulae of section 3.2 by interchanging $x$ and $y$ and replacing the sextupole strength with the skew sextupole strength.

\subsection{Comments}

We have seen in sections 3.1 and 3.2 that by making some assumptions about the machine lattice and the placement of correction elements, the task of determining the effectiveness of a given set of correctors becomes rather straight forward. Here we discuss the conditions under which these assumptions are valid.

Consider first the case in which the lattice is composed of $N$ identical FODO cells, and let $\phi_{x}=\mu_{x} / Q_{x}$ and $\phi_{y}=\mu_{y} / Q_{y}$ be the normalized betatron phase advances in the $x$ and $y$ planes between two horizontal beta maximums, two vertical beta minimums, or between a beta minimum and a beta maximum. Then in each case $\phi_{x}=\phi_{y}$. Furthermore any two horizontal beta maximums (or minimums) in the lattice are equal, any two vertical beta maximums (or minimums) are equal, and the beta maximums in one plane occur at the same locations as the beta minimums in the opposite plane. Thus, if two correction elements are placed at horizontal beta maximums and two at vertical beta maximums, then all of the assumptions made in sections 3.1 and 3.2 are valid.

Now in general not all of the FODO cells in an AGS lattice are identical. However there are usually symmetries which imply that the assumptions 
made in sections 3.1 and 3.2 are valid for some set of points in the lattice. In each superperiod of the Brookhaven AGS, for example, we have

$$
\begin{aligned}
& \beta_{x}\left(s_{5}-t\right)=\beta_{x}\left(s_{5}+t\right)=\beta_{y}\left(s_{15}-t\right)=\beta_{y}\left(s_{15}+t\right) \\
& \beta_{y}\left(s_{5}-t\right)=\beta_{y}\left(s_{5}+t\right)=\beta_{x}\left(s_{15}-t\right)=\beta_{x}\left(s_{15}+t\right)
\end{aligned}
$$

and

$$
\begin{aligned}
& \phi_{x}\left(s_{15}+t\right)-\phi_{x}\left(s_{15}-t\right)=\phi_{y}\left(s_{5}+t\right)-\phi_{y}\left(s_{5}-t\right), \\
& \phi_{x}\left(s_{5}+t\right)-\phi_{x}\left(s_{5}-t\right)=\phi_{y}\left(s_{15}+t\right)-\phi_{y}\left(s_{15}-t\right), \\
& \phi_{x}\left(s_{15}-t\right)-\phi_{x}\left(s_{5}+t\right)=\phi_{y}\left(s_{15}-t\right)-\phi_{y}\left(s_{5}+t\right),
\end{aligned}
$$

where $s_{5}$ and $s_{15}$ are respectively the distances from the beginning of a superperiod to the middle of the number 5 and number 15 straight sections, $0<t<s_{5}$, and $\phi_{x}$ and $\phi_{y}$ are the normalized betatron phase advances defined in equation (18). Adding equations (35) we have also

$$
\phi_{x}\left(s_{15}+t\right)-\phi_{x}\left(s_{5}-t\right)=\phi_{y}\left(s_{15}+t\right)-\phi_{y}\left(s_{5}-t\right) .
$$

Thus in each superperiod we have

$$
\begin{aligned}
\beta_{x 1}=\beta_{x 9}=\beta_{y 11}=\beta_{y 19}, & \beta_{x 3}=\beta_{x 7}=\beta_{y 13}=\beta_{y 17} \\
\beta_{y 1}=\beta_{y 9}=\beta_{x 11}=\beta_{x 19}, & \beta_{y 3}=\beta_{y 7}=\beta_{x 13}=\beta_{x 17} \\
\beta_{x 5}=\beta_{y 15}, & \beta_{y 5}=\beta_{x 15}
\end{aligned}
$$

and

$$
\begin{aligned}
\phi_{x 19}-\phi_{x 1}= & \phi_{y 19}-\phi_{y 1}, \quad \phi_{x 17}-\phi_{x 3}=\phi_{y 17}-\phi_{y 3}, \\
\phi_{x 15}-\phi_{x 5}= & \phi_{y 15}-\phi_{y 5}, \quad \phi_{x 13}-\phi_{x 7}=\phi_{y 13}-\phi_{y 7}, \\
& \phi_{x 11}-\phi_{x 9}=\phi_{y 11}-\phi_{y 9},
\end{aligned}
$$

where the numbers 1-19 correspond to straight sections 1-19. (Note that beta minima and maxima occur only in the odd numbered straight sections of the AGS). It is, of course, also true that $\beta_{x 1}=\beta_{x 2}, \beta_{y 1}=\beta_{y 2}$, and $\phi_{x 2}-\phi_{x 1}=\phi_{y 2}-\phi_{y 1}$ for any two points, 1 and 2, separated by one or more superperiods in the AGS.

The assumptions of sections 3.1 and 3.2 are therefore valid if two correction elements are placed in any one pair of the following pairs of straight sections: $(1,19),(3,17),(5,15),(7,13),(9,11)$, and another two are placed in the same straight sections of another superperiod. 
In addition to the relations $(34-38)$, which are nearly exact, we have the following approximate relations due to the shortening of magnets $1,2,9$, $10,11,12,19$, and 20 in each superperiod [4]:

$$
\begin{aligned}
& \beta_{x 5} \approx \beta_{x 9} \approx \beta_{x 13}, \quad \beta_{y 5} \approx \beta_{y 9} \approx \beta_{y 13}, \\
& \beta_{x 7} \approx \beta_{x 11} \approx \beta_{x 15}, \quad \beta_{y 7} \approx \beta_{y 11} \approx \beta_{y 15} .
\end{aligned}
$$

It follows that the five FODO cells in each superperiod are approximately equivalent so that the results stated above for the case of a lattice composed of identical FODO cells are approximately true. Thus, if correctors are placed at any two horizontal beta maximums and at any two vertical beta maximums in the AGS, then the assumptions of sections 3.1-3.2 are always at least approximately valid and one may use the formulae developed in these sections to estimate the effectiveness of the correctors.

In the AGS Booster each of the six superperiods is composed of four FODO cells which are to first order identical. Therefore, if one places correctors at any two horizontal beta maximums and at any two vertical beta maximums in the booster lattice, the assumptions of sections 3.1-3.2 are valid.

\section{Application to the AGS}

The correction schemes discussed in the following sections (4.1-4.3) were first worked out by $\mathrm{E}$. Raka $[5,6]$. We re-derive his results here using the formulae developed in section 3 .

\subsection{Correction of resonances $2 Q_{x}=17$ and $2 Q_{y}=17$}

In Raka's scheme for the correction of these resonances one first considers four correction quadrupoles located in the C3, F3, C17, and F17 straight sections. We shall take positions $s_{1}, s_{2}, s_{3}, s_{4}$ to be the locations of the quads in straight sections $\mathrm{C} 3, \mathrm{~F} 3, \mathrm{C} 17$, and F17 respectively, with $s_{1}=0$. Then using equations (34-36) and the superperiod symmetry we have

$$
\beta_{x 1}=\beta_{x 2}=\beta_{y 3}=\beta_{y 4}=b, \quad \beta_{y 1}=\beta_{y 2}=\beta_{x 3}=\beta_{x 4}=B
$$

and

$$
\phi_{1}=\phi_{x}(C 3)=\phi_{y}(C 3)=0, \quad \phi_{2}=\phi_{x}(F 3)=\phi_{y}(F 3)
$$




$$
\phi_{3}=\phi_{x}(C 17)=\phi_{y}(C 17), \quad \phi_{4}=\phi_{x}(F 17)=\phi_{y}(F 17) .
$$

Now the normalized betatron phase advance between two points separated by three superperiods in the AGS is $\pi / 2$, and the normalized phase advance between the number 3 and number 17 straight sections of a superperiod is very nearly $2 \pi / 17$. Thus we have

$$
\phi_{1}=0, \quad \phi_{2}=\frac{\pi}{2}, \quad \phi_{3}=\frac{2 \pi}{17}, \quad \phi_{4}=\frac{\pi}{2}+\frac{2 \pi}{17} .
$$

Using (40-42) and $p=17$ in the equations of section 3.1.2 we find that the excitation coefficients produced by the four correctors are

where

$$
\left(\begin{array}{c}
C X_{1} \\
S X_{1} \\
C Y_{1} \\
S Y_{1}
\end{array}\right)=C\left(\frac{e Q}{c P}\right) \mathbf{M}_{1}\left(\begin{array}{c}
I_{1} \\
I_{2} \\
I_{3} \\
I_{4}
\end{array}\right),
$$

$$
\begin{gathered}
\mathbf{M}_{1}=b\left(\begin{array}{rr}
1 & R \\
-R & -1
\end{array}\right)\left(\begin{array}{ll}
\mathbf{I} & \mathbf{0} \\
\mathbf{0} & \mathbf{I}
\end{array}\right), \quad \mathbf{I}=\left(\begin{array}{ll}
1 & 0 \\
0 & 1
\end{array}\right), \\
\mathbf{M}_{1}^{-1}=\frac{1}{b\left(R^{2}-1\right)}\left(\begin{array}{rr}
-1 & -R \\
R & 1
\end{array}\right)\left(\begin{array}{ll}
\mathbf{I} & \mathbf{0} \\
\mathbf{0} & \mathbf{I}
\end{array}\right), \quad R=B / b .
\end{gathered}
$$

Now, to insure that the correction scheme does not introduce any $9 \theta$ harmonic components, additional quadrupoles at $\mathrm{E} 3, \mathrm{H} 3, \mathrm{E} 17, \mathrm{H} 17$ are excited with the same currents as the quads at C3, F3, C17, F17 respectively. Since the additional quads are two superperiods away from the first set of quads, we have

$$
\begin{aligned}
\beta_{x, y}(E 3)=\beta_{x, y}(C 3), & \beta_{x, y}(H 3)=\beta_{x, y}(F 3), \\
\beta_{x, y}(E 17)=\beta_{x, y}(C 17), & \beta_{x, y}(H 17)=\beta_{x, y}(F 17), \\
\phi_{x}(E 3)=\phi_{y}(E 3)=\phi_{1}+\omega, & \phi_{x}(H 3)=\phi_{y}(H 3)=\phi_{2}+\omega, \\
\phi_{x}(E 17)=\phi_{y}(E 17)=\phi_{3}+\omega, & \phi_{x}(H 17)=\phi_{y}(H 17)=\phi_{4}+\omega,
\end{aligned}
$$

where $\phi_{1}-\phi_{4}$ are given by (42), and $\omega=\pi / 3$ is the normalized betatron phase advance for two superperiods. The excitation coefficients produced by the additional quads are therefore

$$
\left(\begin{array}{c}
C X_{2} \\
S X_{2} \\
C Y_{2} \\
S Y_{2}
\end{array}\right)=C\left(\frac{e Q}{c P}\right) \mathrm{M}_{2}\left(\begin{array}{c}
I_{1} \\
I_{2} \\
I_{3} \\
I_{4}
\end{array}\right),
$$


where

$$
\mathbf{M}_{2}=\left(\begin{array}{cc}
\eta & 0 \\
0 & \eta
\end{array}\right) \mathbf{M}_{1}, \quad \eta=\left(\begin{array}{rr}
\cos (p \omega) & -\sin (p \omega) \\
\sin (p \omega) & \cos (p \omega)
\end{array}\right),(p \omega=1.7 \pi / 3)
$$

The excitation coefficients produced by both sets of quadrupoles are given by the sum of equations (43) and (46).

In addition to insuring that no $9 \theta$ harmonic components are introduced by the correction scheme it is necessary to insure that no $0 \theta$ components are introduced. (Any $0 \theta$ components would alter the machine tunes). This is accomplished by adding a second group of eight quadrupoles to the scheme. These eight quads, at I3, L3, I17, L17, K3, B3, K17, and B17, are excited with currents opposite to those in the first group at C3, F3, C17, F17, E3, H3, E17, and H17 respectively. Using the fact that the second group of quads are a normalized betatron phase advance of $\pi$ away from the first group, the equations of section 3.1 .2 with $p=17$ show that the second group produces the same excitation coefficients as the first group. Thus the excitation coefficients produced by all 16 quads are

$$
\left(\begin{array}{c}
C X \\
S X \\
C Y \\
S Y
\end{array}\right)=C\left(\frac{e Q}{c P}\right) \mathrm{M}\left(\begin{array}{c}
I_{1} \\
I_{2} \\
I_{3} \\
I_{4}
\end{array}\right)
$$

where

$$
\begin{gathered}
\mathbf{M}=2\left(\mathbf{M}_{1}+\mathbf{M}_{2}\right)=2\left(\begin{array}{ll}
\mathbf{\Omega} & \mathbf{0} \\
\mathbf{0} & \mathbf{\Omega}
\end{array}\right) \mathbf{M}_{1}, \\
\mathbf{M}^{-1}=\frac{1}{2} \mathbf{M}_{1}^{-1}\left(\begin{array}{ll}
\mathbf{\Omega}^{-1} & \mathbf{0} \\
\mathbf{0} & \mathbf{\Omega}^{-1}
\end{array}\right), \\
\mathbf{\Omega}=\mathbf{I}+\eta=\frac{1}{2}\left(\begin{array}{rr}
3 & \sqrt{3} \\
-\sqrt{3} & 3
\end{array}\right), \quad \mathbf{\Omega}^{-1}=\frac{2}{12}\left(\begin{array}{rr}
3 & -\sqrt{3} \\
\sqrt{3} & 3
\end{array}\right) .
\end{gathered}
$$

Using (44-45) in (49) we then have

$$
\mathbf{M}=2 b\left(\begin{array}{rr}
\Omega & R \Omega \\
-R \Omega & -\Omega
\end{array}\right)=b\left(\begin{array}{rrrr}
3 & \sqrt{3} & 3 R & \sqrt{3} R \\
-\sqrt{3} & 3 & -\sqrt{3} R & 3 R \\
-3 R & -\sqrt{3} R & -3 & -\sqrt{3} \\
\sqrt{3} R & -3 R & \sqrt{3} & -3
\end{array}\right)
$$




$$
\mathbf{M}^{-1}=\frac{1}{12 b\left(R^{2}-1\right)}\left(\begin{array}{rrrr}
-3 & \sqrt{3} & -3 R & \sqrt{3} R \\
-\sqrt{3} & -3 & -\sqrt{3} R & -3 R \\
3 R & -\sqrt{3} R & 3 & -\sqrt{3} \\
\sqrt{3} R & 3 R & \sqrt{3} & 3
\end{array}\right) .
$$

The currents which produce the desired corrections are then

$$
\left(\begin{array}{c}
I_{1} \\
I_{2} \\
I_{3} \\
I_{4}
\end{array}\right)=\frac{1}{C}\left(\frac{c P}{e Q}\right) \mathbf{M}^{-1}\left(\begin{array}{c}
C X \\
S X \\
C Y \\
S Y
\end{array}\right)
$$

\subsection{Correction of resonances $3 Q_{x}=26$ and $Q_{x}+2 Q_{y}=26$}

In Raka's scheme $[5,6]$ for the correction of these resonances one first considers four correction sextupoles located in the $\mathrm{C} 7, \mathrm{E} 7, \mathrm{C} 13$, and $\mathrm{E} 13$ straight sections. We shall take positions $s_{1}, s_{2}, s_{3}, s_{4}$ to be the locations of the sextupoles in straight sections $\mathrm{C} 7, \mathrm{E} 7, \mathrm{C} 13$, and $\mathrm{E} 13$ respectively, with $s_{1}=0$. Then using equations (34-36) and the superperiod symmetry we have

$$
\beta_{x 1}=\beta_{x 2}=\beta_{y 3}=\beta_{y 4}=b, \quad \beta_{y 1}=\dot{\beta}_{y 2}=\beta_{x 3}=\beta_{x 4}=B,
$$

and

$$
\begin{aligned}
& \phi_{1}=\phi_{x}(C 7)=\phi_{y}(C 7)=0, \quad \phi_{2}=\phi_{x}(E 7)=\phi_{y}(E 7) \\
& \phi_{3}=\phi_{x}(C 13)=\phi_{y}(C 13), \quad \phi_{4}=\phi_{x}(E 13)=\phi_{y}(E 13) .
\end{aligned}
$$

Now the normalized betatron phase advance between two points separated by two superperiods in the AGS is $\pi / 3$, and the normalized phase advance between the number 7 and number 13 straight sections of a superperiod is $\phi \approx \pi / 20$. Thus we have

$$
\phi_{1}=0, \quad \phi_{2}=\pi / 3, \quad \phi_{3}=\phi, \quad \phi_{4}=\pi / 3+\phi
$$

Using (53-55) and $p=26$ in the equations of section 3.2 we find that the excitation coefficients produced by the four correctors are

$$
\left(\begin{array}{c}
C X_{1} \\
S X_{1} \\
C Y_{1} \\
S Y_{1}
\end{array}\right)=C\left(\frac{e S}{c P}\right) \mathbf{M}\left(\begin{array}{c}
I_{1} \\
I_{2} \\
I_{3} \\
I_{4}
\end{array}\right)
$$


where

$$
\mathbf{M}=b^{3 / 2}\left(\begin{array}{rrrr}
C_{1} & C_{2} & R^{3 / 2} C_{3} & R^{3 / 2} C_{4} \\
S_{1} & S_{2} & R^{3 / 2} S_{3} & R^{3 / 2} S_{4} \\
-R C_{1} & -R C_{2} & -R^{1 / 2} C_{3} & -R^{1 / 2} C_{4} \\
-R S_{1} & -R S_{2} & -R^{1 / 2} S_{3} & -R^{1 / 2} S_{4}
\end{array}\right)
$$

and

$$
\begin{aligned}
& \mathbf{M}^{-1}=G b^{-3 / 2}\left(\begin{array}{rrrr}
-R^{1 / 2} S_{2} & R^{1 / 2} C_{2} & -R^{3 / 2} S_{2} & R^{3 / 2} C_{2} \\
R^{1 / 2} S_{1} & -R^{1 / 2} C_{1} & R^{3 / 2} S_{1} & -R^{3 / 2} C_{1} \\
R S_{4} & -R C_{4} & S_{4} & -C_{4} \\
-R S_{3} & R C_{3} & -S_{3} & C_{3}
\end{array}\right) \text {, } \\
& R=B / b, \quad G=\frac{1}{R^{1 / 2}\left(R^{2}-1\right)} \frac{2}{\sqrt{3}} \\
& C_{1}=1, \quad C_{2}=-1 / 2, \quad C_{3}=\cos \omega, \quad C_{4}=\cos (\omega+2 \pi / 3), \\
& S_{1}=0, \quad S_{2}=\sqrt{3} / 2, \quad S_{3}=\sin \omega, \quad S_{4}=\sin (\omega+2 \pi / 3),
\end{aligned}
$$

and $\omega=26 \phi \approx 26 \pi / 20$.

Now, to insure that the correction scheme does not introduce any $0 \theta$ harmonic components-which would alter the machine chromaticities-additional sextupoles at $\mathrm{F} 7, \mathrm{H} 7, \mathrm{~F} 13$, and $\mathrm{H} 13$ are excited with currents opposite to those at $\mathrm{C} 7, \mathrm{E} 7, \mathrm{C} 13$, and E13 respectively. Since the additional sextupoles are three superperiods away from the first set of sextupoles, the normalized betatron phase advance between the two sets is $\pi / 2$, and hence with $p=26$ the equations of section 3.2 show that the additional set produces the same excitation coefficients as the first set. To insure that no odd harmonics in $\theta$, and in particular no $9 \theta$ or $17 \theta$ harmonics, are produced, a second group of eight sextupoles is added to the scheme. These eight sextupoles, at I7, K7, I13, K13, L7, B7, L13, and $\mathrm{B} 13$ are excited with the same currents as those at $\mathrm{C} 7, \mathrm{E} 7, \mathrm{C} 13, \mathrm{E} 13, \mathrm{~F} 7$, $\mathrm{H} 7, \mathrm{~F} 13$, and $\mathrm{H13}$ respectively. Using the fact that the second group of sextupoles is a normalized betatron phase advance of $\pi$ away from the first group, the equations of section 3.2 with $p=26$ show that the second group produces the same excitation coefficients as the first group. Thus the excitation coefficients produced by all 16 sextupoles are four times those produced by the original set of four, and are therefore

$$
\left(\begin{array}{c}
C X \\
S X \\
C Y \\
S Y
\end{array}\right)=4 C\left(\frac{e S}{c P}\right) \mathrm{M}\left(\begin{array}{c}
I_{1} \\
I_{2} \\
I_{3} \\
I_{4}
\end{array}\right)
$$


where $\mathbf{M}$ is given by (57). The currents which produce the desired corrections are then

$$
\left(\begin{array}{c}
I_{1} \\
I_{2} \\
I_{3} \\
I_{4}
\end{array}\right)=\frac{1}{4 C}\left(\frac{c P}{e S}\right) \mathbf{M}^{-1}\left(\begin{array}{c}
C X \\
S X \\
C Y \\
S Y
\end{array}\right)
$$

where $\mathrm{M}^{-1}$ is given by (58).

\subsection{Correction of resonances $3 Q_{y}=26$ and $2 Q_{x}+Q_{y}=26$}

These resonances are currently corrected with four air-core skew sextupoles located in straight sections E15, F15, I5, and K5. However, during the 1989 summer shutdown the skew sextupole in E15 will be removed and it will no longer be possible to correct both resonances simultaneously. During the 1990 summer shutdown the remaining air-core skew sextupoles will be removed and four new iron-core units will be installed. A number of correction schemes using these new skew sextupoles have been considered, and based on constraints imposed by the straight section committee (Willem van Asselt) and the vacuum group (Kimo Welch) two sets of locations for the magnets are currently recognized as possibilities. One set of locations would consist of straight sections 7 and 13 in one superperiod and the same straight sections two superperiods away. The other set would consist of straight sections 1 and 19 in one superperiod and the same straight sections two superperiods away. In each case the two pairs of straight sections are separated by two superperiods so that additional magnets may be added, (if necessary) as in the scheme discussed in the previous section, to insure that no harmful harmonic components are produced.

Here we consider the more general case in which skew sextupoles are placed in straight sections $i$ and $j$ of superperiod $M$ and in the same straight sections of superperiod $N$, where $(i, j)$ is any one of the pairs $(19$, $1),(3,17),(15,5),(7,13)$, or $(11,9)$, and $M$ and $N$ are $n$ superperiods apart. (Note that the first number of each pair corresponds to a vertical beta maximum and the second to a horizontal beta maximum). We shall take positions $s_{1}, s_{2}, s_{3}$, and $s_{4}$ to be the locations of the skew sextupoles in straight sections $\mathrm{Mi}, \mathrm{Ni}, \mathrm{Mj}$, and $\mathrm{Nj}$ respectively, with $s_{1}=0$. Then 
using equations (34-36) and the superperiod symmetry we have

$$
\beta_{x 1}=\beta_{x 2}=\beta_{y 3}=\beta_{y 4}=b, \quad \beta_{y 1}=\beta_{y 2}=\beta_{x 3}=\beta_{x 4}=B
$$

and

$$
\begin{gathered}
\phi_{1}=\phi_{x}(M i)=\phi_{y}(M i)=0, \quad \phi_{2}=\phi_{x}(N i)=\phi_{y}(N i), \\
\phi_{3}=\phi_{x}(M j)=\phi_{y}(M j), \quad \phi_{4}=\phi_{x}(N j)=\phi_{y}(N j) .
\end{gathered}
$$

Now the normalized betatron phase advance between two points separated by $n$ superperiods in the AGS is $n \pi / 6$, and the normalized phase advance between the $i$ and $j$ straight sections of a superperiod is $\phi \approx(j-i) \pi / 120$.

Thus we have

$$
\phi_{1}=0, \quad \phi_{2}=n \pi / 6, \quad \phi_{3}=\phi, \quad \phi_{4}=\phi+n \pi / 6 .
$$

Using (61-63) and $p=26$ in the equations of section 3.2 with $x$ and $y$ interchanged we find that the excitation coefficients produced by the four correctors are

$$
\left(\begin{array}{c}
C Y \\
S Y \\
C X \\
S X
\end{array}\right)=C\left(\frac{e S}{c P}\right) \mathrm{M}\left(\begin{array}{c}
I_{1} \\
I_{2} \\
I_{3} \\
I_{4}
\end{array}\right)
$$

where $C Y$ and $S Y$ are the cos and sin parts of $\kappa_{y}, C X$ and $S X$ are the cos and sin parts of $\kappa_{y x} / 3$, and

$$
\mathbf{M}=b^{3 / 2}\left(\begin{array}{rrrr}
R^{3 / 2} C_{1} & R^{3 / 2} C_{2} & C_{3} & C_{4} \\
R^{3 / 2} S_{1} & R^{3 / 2} S_{2} & S_{3} & S_{4} \\
-R^{1 / 2} C_{1} & -R^{1 / 2} C_{2} & -R C_{3} & -R C_{4} \\
-R^{1 / 2} S_{1} & -R^{1 / 2} S_{2} & -R S_{3} & -R S_{4}
\end{array}\right)
$$

and

$$
\begin{gathered}
\mathbf{M}^{-1}=G b^{-3 / 2}\left(\begin{array}{rrrr}
-R S_{2} & R C_{2} & -S_{2} & C_{2} \\
R S_{1} & -R C_{1} & S_{1} & -C_{1} \\
R^{1 / 2} S_{4} & -R^{1 / 2} C_{4} & R^{3 / 2} S_{4} & -R^{3 / 2} C_{4} \\
-R^{1 / 2} S_{3} & R^{1 / 2} C_{3} & -R^{3 / 2} S_{3} & R^{3 / 2} C_{3}
\end{array}\right), \\
R=B / 6, \quad G=\frac{-1}{R^{1 / 2}\left(R^{2}-1\right) \sin (n \pi / 3)}, \\
C_{1}=1, \quad C_{2}=\cos (n \pi / 3), \quad C_{3}=\cos \omega, \quad C_{4}=\cos (\omega+n \pi / 3),
\end{gathered}
$$




$$
S_{1}=0, \quad S_{2}=\sin (n \pi / 3), \quad S_{3}=\sin \omega, \quad S_{4}=\sin (\omega+n \pi / 3),
$$

and $\omega=26 \phi \approx 26(j-i) \pi / 120$. The currents which produce the desired corrections are then

$$
\left(\begin{array}{c}
I_{1} \\
I_{2} \\
I_{3} \\
I_{4}
\end{array}\right)=\frac{1}{C}\left(\frac{c P}{e S}\right) \mathbf{M}^{-1}\left(\begin{array}{c}
C Y \\
S Y \\
C X \\
S X
\end{array}\right)
$$

where $\mathbf{M}^{-1}$ is given by (66).

\section{Application to the Booster}

The booster lattice [7] consists of six superperiods-labled A, B, C, D, E, and $\mathrm{F}$-each containing four FODO cells which are, to first order, identical. The positions of the horizontal beta maximums in each superperiod are $s_{1}, s_{3}, s_{5}, s_{7}$, and those of the vertical beta maximums are $s_{0}, s_{2}, s_{4}, s_{6}, s_{8}$, with $s_{j}>s_{i}$ if $j>i$. We shall take $s_{0}$ in superperiod A to be the point of zero betatron phase. The normalized betatron phase advance (defined by equation 18 ) for each superperiod is $\pi / 3$. Assuming the four FODO cells in each superperiod are identical we have

$$
\phi_{i}=\phi_{x i}=\phi_{y i}=i \pi / 24
$$

and

$$
\begin{gathered}
\beta_{x 0}=\beta_{x 2}=\beta_{x 4}=\beta_{x 6}=\beta_{x 8}=a, \\
\beta_{y 0}=\beta_{y 2}=\beta_{y 4}=\beta_{y 6}=\beta_{y 8}=B, \\
\beta_{x 1}=\beta_{x 3}=\beta_{x 5}=\beta_{x 7}=A, \\
\beta_{y 1}=\beta_{y 3}=\beta_{y 5}=\beta_{y 7}=b,
\end{gathered}
$$

where $\phi_{x i}$ and $\phi_{y i}$ are the normalized betatron phase advances at the positions $s_{i}$, and $\beta_{x i}=\beta_{x}\left(s_{i}\right), \beta_{y i}=\beta_{y}\left(s_{i}\right)$. To first order we also have

$$
a=b, \quad A=B .
$$

Although equations (68-70) are not exact-because the booster dipoles do not occupy the same positions in each FODO cell-they are good enough 
for estimating the currents required for various correction schemes in the booster and therefore will be used in the following sections.

The correction elements for the schemes discussed in the following sections are located at or near the positions $s_{i}$ in each superperiod and are excited with currents $I_{j i}$ where $j=1,2,3,4,5$, and 6 corresponds to superperiods $\mathrm{A}, \mathrm{B}, \mathrm{C}, \mathrm{D}, \mathrm{E}$, and $\mathrm{F}$ respectively. Tepikian has shown $[8,9]$ that by choosing

$$
I_{j i}=f_{j} I_{i}, \quad f_{j}=\cos [p(j-1) \pi / 3],
$$

one can correct the $m Q_{x}+n Q_{y}=p$ resonances without introducing unwanted harmonics.

\subsection{Correction of resonances $2 Q_{x}=9$ and $2 Q_{y}=9$}

Using equation (68) we find that for $p=9$ the phase differences, $p \phi_{6}-p \phi_{2}$ and $p \phi_{5}-p \phi_{1}$ are odd multiples of $\pi / 2$ which, as we have shown in section 3 , gives the most effective correction of the resonances. Thus, for the correction of the $2 Q_{x}=9$ and $2 Q_{y}=9$ resonances we consider the four correction quadrupoles located in superperiod $A$ at $s_{1}, s_{2}, s_{5}$, and $s_{6}$. Using (68-70) and $p=9$ in the equations of section 3.1 .2 we find that the excitation coefficients produced by the four correctors are

$$
\left(\begin{array}{c}
C X_{a} \\
S X_{a} \\
C Y_{a} \\
S Y_{a}
\end{array}\right)=C\left(\frac{e Q}{c P}\right) \mathbf{M}_{a}\left(\begin{array}{c}
I_{2} \\
I_{6} \\
I_{1} \\
I_{5}
\end{array}\right)
$$

where

$$
\begin{gathered}
\mathbf{M}_{a}=b\left(\begin{array}{rrrr}
C_{2} & C_{6} & R C_{1} & R C_{5} \\
S_{2} & S_{6} & R S_{1} & R S_{5} \\
-R C_{2} & -R C_{6} & -C_{1} & -C_{5} \\
-R S_{2} & -R S_{6} & -S_{1} & -S_{5}
\end{array}\right), \\
C_{j}=\cos (3 \pi j / 8), \quad S_{j}=\sin (3 \pi j / 8), \quad R=B / b,
\end{gathered}
$$

and

$$
\mathbf{M}_{a}^{-1}=\frac{1}{b\left(R^{2}-1\right)}\left(\begin{array}{rrrr}
S_{6} & -C_{6} & R S_{6} & -R C_{6} \\
-S_{2} & C_{2} & -R S_{2} & R C_{2} \\
-R S_{5} & R C_{5} & -S_{5} & C_{5} \\
R S_{1} & -R C_{1} & S_{1} & -C_{1}
\end{array}\right)
$$


If we now excite the correctors at $s_{8}, s_{4}, s_{7}$, and $s_{3}$ with currents $I_{8}=I_{2}$, $I_{4}=-I_{6}, I_{7}=I_{1}$, and $I_{3}=-I_{5}$ we find that the excitation coefficients produced by these quadrupoles are

$$
\left(\begin{array}{c}
C X_{b} \\
S X_{b} \\
C Y_{b} \\
S Y_{b}
\end{array}\right)=C\left(\frac{e Q}{c P}\right) \mathrm{M}_{b}\left(\begin{array}{c}
I_{2} \\
I_{6} \\
I_{1} \\
I_{5}
\end{array}\right)
$$

where

$$
\mathbf{M}_{b}=\left(\begin{array}{cc}
\eta & 0 \\
0 & \eta
\end{array}\right) \mathbf{M}_{a}, \quad \eta=\left(\begin{array}{rr}
\cos (\pi / 4) & -\sin (\pi / 4) \\
\sin (\pi / 4) & \cos (\pi / 4)
\end{array}\right)
$$

The excitation coefficients produced by all eight quadrupoles in superperiod $A$ are then

$$
\left(\begin{array}{c}
C X_{1} \\
S X_{1} \\
C Y_{1} \\
S Y_{1}
\end{array}\right)=C\left(\frac{e Q}{c P}\right) \mathbf{M}_{1}\left(\begin{array}{c}
I_{2} \\
I_{6} \\
I_{1} \\
I_{5}
\end{array}\right)
$$

where

$$
\begin{gathered}
\mathbf{M}_{1}=\mathbf{M}_{a}+\mathbf{M}_{b}=\left(\begin{array}{cc}
\mathbf{\Omega} & 0 \\
\mathbf{0} & \mathbf{\Omega}
\end{array}\right) \mathbf{M}_{a} \\
\mathbf{\Omega}=\left(\begin{array}{cc}
1+\cos (\pi / 4) & -\sin (\pi / 4) \\
\sin (\pi / 4) & 1+\cos (\pi / 4)
\end{array}\right)=\frac{1}{2}\left(\begin{array}{cc}
2+\sqrt{2} & -\sqrt{2} \\
\sqrt{2} & 2+\sqrt{2}
\end{array}\right) .
\end{gathered}
$$

Now, in the scheme proposed by S. Tepikian [8] for the correction of the $2 Q_{x}=9$ and $2 Q_{y}=9$ resonances, the quadrupoles in the remaining superperiods are excited with currents given by (71). This insures that no $10 \theta, 5 \theta, 4 \theta$, or $0 \theta$ harmonic components are produced by the scheme. The excitation coefficients produced by the quadrupoles in superperiod $j$ are then

$$
\left(\begin{array}{c}
C X_{j} \\
S X_{j} \\
C Y_{j} \\
S Y_{j}
\end{array}\right)=C\left(\frac{e Q}{c P}\right) f_{j} \mathbf{M}_{j}\left(\begin{array}{c}
I_{2} \\
I_{6} \\
I_{1} \\
I_{5}
\end{array}\right)
$$

where

$$
\mathbf{M}_{j}=\left(\begin{array}{ll}
\eta_{j} & \mathbf{0} \\
\mathbf{0} & \eta_{j}
\end{array}\right) \mathbf{M}_{1}, \quad \eta_{j}=\left(\begin{array}{rr}
\cos p(j-1) \pi / 3 & -\sin p(j-1) \pi / 3 \\
\sin p(j-1) \pi / 3 & \cos p(j-1) \pi / 3
\end{array}\right)
$$


and $\mathrm{M}_{1}$ is given by (78) and (73). Summing equation (79) over $j$ we find that the excitation coefficients produced by the quadrupoles in all six superperiods are

$$
\left(\begin{array}{c}
C X \\
S X \\
C Y \\
S Y
\end{array}\right)=C\left(\frac{e Q}{c P}\right) \mathbf{M}\left(\begin{array}{c}
I_{2} \\
I_{6} \\
I_{1} \\
I_{5}
\end{array}\right)
$$

where, for $p=9$,

$$
\mathbf{M}=\sum_{j=1}^{6} f_{j} \mathbf{M}_{j}=6 \mathbf{M}_{1}
$$

The currents which produce the desired corrections are then

$$
\left(\begin{array}{c}
I_{2} \\
I_{6} \\
I_{1} \\
I_{5}
\end{array}\right)=\frac{1}{C}\left(\frac{c P}{e Q}\right) \mathbf{M}^{-1}\left(\begin{array}{c}
C X \\
S X \\
C Y \\
S Y
\end{array}\right)
$$

\subsection{Correction of resonances $3 Q_{x}=14$ and $Q_{x}+2 Q_{y}=14$}

Using equation (68) we find that for $p=14$ the phase differences, $p \phi_{8}-p \phi_{2}$ and $p \phi_{7}-p \phi_{1}$ are odd multiples of $\pi / 2$ which, as we have shown in section 3.2 , gives the most effective correction of the resonances. Thus for the correction of the $3 Q_{x}=14$ and $Q_{x}+2 Q_{y}=14$ resonances we consider the four correction sextupoles located in superperiod $\mathrm{A}$ at $s_{1}, s_{2}$, $s_{7}$, and $s_{8}$. Using $(68-70)$ and $p=14$ in the equations of section 3.2 we find that the excitation coefficients produced by the four correctors are

$$
\left(\begin{array}{c}
C X_{a} \\
S X_{a} \\
C Y_{a} \\
S Y_{a}
\end{array}\right)=C\left(\frac{e S}{c P}\right) \mathbf{M}_{a}\left(\begin{array}{c}
I_{2} \\
I_{8} \\
I_{1} \\
I_{7}
\end{array}\right)
$$

where

$$
\mathbf{M}_{a}=b^{3 / 2}\left(\begin{array}{rrrr}
C_{2} & C_{8} & R^{3 / 2} C_{1} & R^{3 / 2} C_{7} \\
S_{2} & S_{8} & R^{3 / 2} S_{1} & R^{3 / 2} S_{7} \\
-R C_{2} & -R C_{8} & -R^{1 / 2} C_{1} & -R^{1 / 2} C_{7} \\
-R S_{2} & -R S_{8} & -R^{1 / 2} S_{1} & -R^{1 / 2} S_{7}
\end{array}\right)
$$


and

$$
R=B / b, \quad C_{j}=\cos (7 \pi j / 12), \quad S_{j}=\sin (7 \pi j / 12) .
$$

If we now excite the correctors at $s_{4}, s_{6}, s_{3}$, and $s_{5}$ with currents $I_{4}=-I_{2}$, $I_{6}=-I_{8}, I_{3}=-I_{1}$, and $I_{5}=-I_{7}$ we find that the excitation coefficients produced by these sextupoles are

$$
\left(\begin{array}{c}
C X_{b} \\
S X_{b} \\
C Y_{b} \\
S Y_{b}
\end{array}\right)=C\left(\frac{e S}{c P}\right) \mathbf{M}_{b}\left(\begin{array}{c}
I_{2} \\
I_{8} \\
I_{1} \\
I_{7}
\end{array}\right)
$$

where

$$
\mathbf{M}_{b}=-b^{3 / 2}\left(\begin{array}{rrrr}
C_{4} & C_{6} & R^{3 / 2} C_{3} & R^{3 / 2} C_{5} \\
S_{4} & S_{6} & R^{3 / 2} S_{3} & R^{3 / 2} S_{5} \\
-R C_{4} & -R C_{6} & -R^{1 / 2} C_{3} & -R^{1 / 2} C_{5} \\
-R S_{4} & -R S_{6} & -R^{1 / 2} S_{3} & -R^{1 / 2} S_{5}
\end{array}\right)
$$

The excitation coefficients produced by all eight sextupoles in superperiod A are then

$$
\left(\begin{array}{c}
C X_{1} \\
S X_{1} \\
C Y_{1} \\
S Y_{1}
\end{array}\right)=C\left(\frac{e S}{c P}\right) \mathbf{M}_{1}\left(\begin{array}{c}
I_{2} \\
I_{8} \\
I_{1} \\
I_{7}
\end{array}\right)
$$

where

$$
\begin{gathered}
\mathbf{M}_{1}=\mathbf{M}_{a}+\mathbf{M}_{b} \\
\mathbf{M}_{1}=2 b^{3 / 2} \cos (\pi / 12)\left(\begin{array}{rrrr}
C_{2}^{+} & C_{8}^{-} & R^{3 / 2} C_{1}^{+} & R^{3 / 2} C_{7}^{-} \\
S_{2}^{+} & S_{8}^{-} & R^{3 / 2} S_{1}^{+} & R^{3 / 2} S_{7}^{-} \\
-R C_{2}^{+} & -R C_{8}^{-} & -R^{1 / 2} C_{1}^{+} & -R^{1 / 2} C_{7}^{-} \\
-R S_{2}^{+} & -R S_{8}^{-} & -R^{1 / 2} S_{1}^{+} & -R^{1 / 2} S_{7}^{-}
\end{array}\right) \\
C_{j}^{+}=\cos \left(p \phi_{j}+\pi / 12\right), \quad S_{j}^{+}=\sin \left(p \phi_{j}+\pi / 12\right) \\
C_{j}^{-}=\cos \left(p \phi_{j}-\pi / 12\right), \quad S_{j}^{-}=\sin \left(p \phi_{j}-\pi / 12\right) \\
\left(p \phi_{j}=14 \phi_{j}=7 \pi j / 12\right)
\end{gathered}
$$

Now, in the scheme proposed by S. Tepikian [9] for the correction of the $3 Q_{x}=p$ and $Q_{x}+2 Q_{y}=p$ resonances, the sextupoles in the remaining superperiods are excited with currents given by (71). For $p=14$ this 
insures that no $13 \theta, 9 \theta, 5 \theta$, or $0 \theta$ harmonics are produced. (The scheme does produce $10 \theta$ and $4 \theta$ harmonic components which are potentially harmful. However, for the tune spreads and operating point expected in the booster this should not be a problem). The excitation coefficients produced by the sextupoles in superperiod $j$ are then

$$
\left(\begin{array}{c}
C X_{j} \\
S X_{j} \\
C Y_{j} \\
S Y_{j}
\end{array}\right)=C\left(\frac{e S}{c P}\right) f_{j} \mathbf{M}_{j}\left(\begin{array}{c}
I_{2} \\
I_{8} \\
I_{1} \\
I_{7}
\end{array}\right)
$$

where

$$
\mathbf{M}_{j}=\left(\begin{array}{cc}
\eta_{j} & 0 \\
\mathbf{0} & \eta_{j}
\end{array}\right) \mathbf{M}_{1}, \quad \eta_{j}=\left(\begin{array}{rr}
\cos p(j-1) \pi / 3 & -\sin p(j-1) \pi / 3 \\
\sin p(j-1) \pi / 3 & \cos p(j-1) \pi / 3
\end{array}\right)
$$

and $\mathbf{M}_{1}$ is given by (88). Summing equation (89) over $j$ we find that the excitation coefficients produced by the sextupoles in all six superperiods are

$$
\left(\begin{array}{c}
C X \\
S X \\
C Y \\
S Y
\end{array}\right)=C\left(\frac{e S}{c P}\right) \mathbf{M}\left(\begin{array}{c}
I_{2} \\
I_{8} \\
I_{1} \\
I_{7}
\end{array}\right)
$$

where, for $p=14$,

$$
\mathbf{M}=\sum_{j=1}^{6} f_{j} \mathbf{M}_{j}=3 \mathbf{M}_{1}
$$

The currents which produce the desired corrections are then

$$
\left(\begin{array}{c}
I_{2} \\
I_{8} \\
I_{1} \\
I_{7}
\end{array}\right)=\frac{1}{C}\left(\frac{c P}{e S}\right) \mathbf{M}^{-1}\left(\begin{array}{c}
C X \\
S X \\
C Y \\
S Y
\end{array}\right)
$$

\subsection{Correction of resonances $3 Q_{x}=13$ and $Q_{x}+2 Q_{y}=13$}

For the correction of these resonances we again consider the four correction sextupoles located in superperiod $\mathrm{A}$ at $s_{1}, s_{2}, s_{7}$, and $s_{8}$. Using (68-70) 
and $p=13$ in the equations of section 3.2 we find that the excitation coefficients produced by the four correctors are

$$
\left(\begin{array}{c}
C X_{a} \\
S X_{a} \\
C Y_{a} \\
S Y_{a}
\end{array}\right)=C\left(\frac{e S}{c P}\right) \mathbf{M}_{a}\left(\begin{array}{c}
I_{2} \\
I_{8} \\
I_{1} \\
I_{7}
\end{array}\right)
$$

where

$$
\mathbf{M}_{a}=b^{3 / 2}\left(\begin{array}{rrrr}
C_{2} & C_{8} & R^{3 / 2} C_{1} & R^{3 / 2} C_{7} \\
S_{2} & S_{8} & R^{3 / 2} S_{1} & R^{3 / 2} S_{7} \\
-R C_{2} & -R C_{8} & -R^{1 / 2} C_{1} & -R^{1 / 2} C_{7} \\
-R S_{2} & -R S_{8} & -R^{1 / 2} S_{1} & -R^{1 / 2} S_{7}
\end{array}\right)
$$

and

$$
R=B / b, \quad C_{j}=\cos (13 \pi j / 24), \quad S_{j}=\sin (13 \pi j / 24) .
$$

(Note that for $p=13$ the phase differences, $p \phi_{8}-p \phi_{2}$ and $p \phi_{7}-p \phi_{1}$, are $2 \pi+5 \pi / 4$. The effectiveness of the correctors is proportional to the $\sin$ of this phase, as discussed in section 3.2).

Now, as before, we excite the correctors at $s_{4}, s_{6}, s_{3}$, and $s_{5}$ with currents $I_{4}=-I_{2}, I_{6}=-I_{8}, I_{3}=-I_{1}$, and $I_{5}=-I_{7}$. The excitation coefficients produced by these sextupoles are

$$
\left(\begin{array}{c}
C X_{b} \\
S X_{b} \\
C Y_{b} \\
S Y_{b}
\end{array}\right)=C\left(\frac{e S}{c P}\right) \mathbf{M}_{b}\left(\begin{array}{c}
I_{2} \\
I_{8} \\
I_{1} \\
I_{7}
\end{array}\right)
$$

where

$$
\mathbf{M}_{b}=-b^{3 / 2}\left(\begin{array}{rrrr}
C_{4} & C_{6} & R^{3 / 2} C_{3} & R^{3 / 2} C_{5} \\
S_{4} & S_{6} & R^{3 / 2} S_{3} & R^{3 / 2} S_{5} \\
-R C_{4} & -R C_{6} & -R^{1 / 2} C_{3} & -R^{1 / 2} C_{5} \\
-R S_{4} & -R S_{6} & -R^{1 / 2} S_{3} & -R^{1 / 2} S_{5}
\end{array}\right)
$$

The excitation coefficients produced by all eight sextupoles in superperiod A are then

$$
\left(\begin{array}{c}
C X_{1} \\
S X_{1} \\
C Y_{1} \\
S Y_{1}
\end{array}\right)=C\left(\frac{e S}{c P}\right) \mathrm{M}_{1}\left(\begin{array}{c}
I_{2} \\
I_{8} \\
I_{1} \\
I_{7}
\end{array}\right)
$$


where

$$
\begin{aligned}
& \mathbf{M}_{1}=\mathbf{M}_{a}+\mathbf{M}_{b} \\
& \mathbf{M}_{1}=2 b^{3 / 2} \cos (\pi / 24)\left(\begin{array}{rrrr}
C_{2}^{+} & C_{8}^{-} & R^{3 / 2} C_{1}^{+} & R^{3 / 2} C_{7}^{-} \\
S_{2}^{+} & S_{8}^{-} & R^{3 / 2} S_{1}^{+} & R^{3 / 2} S_{7}^{-} \\
-R C_{2}^{+} & -R C_{8}^{-} & -R^{1 / 2} C_{1}^{+} & -R^{1 / 2} C_{7}^{-} \\
-R S_{2}^{+} & -R S_{8}^{-} & -R^{1 / 2} S_{1}^{+} & -R^{1 / 2} S_{7}^{-}
\end{array}\right) \text {, } \\
& C_{j}^{+}=\cos \left(p \phi_{j}+\pi / 24\right), \quad S_{j}^{+}=\sin \left(p \phi_{j}+\pi / 24\right), \\
& C_{j}^{-}=\cos \left(p \phi_{j}-\pi / 24\right), \quad S_{j}^{-}=\sin \left(p \phi_{j}-\pi / 24\right), \\
& \left(p \phi_{j}=13 \phi_{j}=13 \pi j / 24\right) \text {. }
\end{aligned}
$$

Now, as before, we excite the sextupoles in the remaining superperiods with currents given by (71). For $p=13$ this insures that no $14 \theta, 10 \theta, 9 \theta$, $4 \theta$, or $0 \theta$ harmonics are produced. (The scheme does produce $5 \theta$ harmonic components which are potentially harmful. However, for the tune spreads and operating point expected in the booster this should not be a problem). The excitation coefficients produced by the sextupoles in superperiod $j$ are then

$$
\left(\begin{array}{c}
C X_{j} \\
S X_{j} \\
C Y_{j} \\
S Y_{j}
\end{array}\right)=C\left(\frac{e S}{c P}\right) f_{j} \mathbf{M}_{j}\left(\begin{array}{c}
I_{2} \\
I_{8} \\
I_{1} \\
I_{7}
\end{array}\right)
$$

where

$$
\mathbf{M}_{j}=\left(\begin{array}{cc}
\eta_{j} & 0 \\
\mathbf{0} & \eta_{j}
\end{array}\right) \mathbf{M}_{1}, \quad \eta_{j}=\left(\begin{array}{rr}
\cos p(j-1) \pi / 3 & -\sin p(j-1) \pi / 3 \\
\sin p(j-1) \pi / 3 & \cos p(j-1) \pi / 3
\end{array}\right)
$$

and $\mathbf{M}_{1}$ is given by (97). Summing equation (98) over $j$ we find that the excitation coefficients produced by the sextupoles in all six superperiods are

$$
\left(\begin{array}{c}
C X \\
S X \\
C Y \\
S Y
\end{array}\right)=C\left(\frac{e S}{c P}\right) \mathbf{M}\left(\begin{array}{c}
I_{2} \\
I_{8} \\
I_{1} \\
I_{7}
\end{array}\right)
$$

where, for $p=13$,

$$
\mathbf{M}=\sum_{j=1}^{6} f_{j} \mathbf{M}_{j}=3 \mathbf{M}_{1}
$$


The currents which produce the desired corrections are then

$$
\left(\begin{array}{c}
I_{2} \\
I_{8} \\
I_{1} \\
I_{7}
\end{array}\right)=\frac{1}{C}\left(\frac{c P}{e S}\right) \mathbf{M}^{-1}\left(\begin{array}{c}
C X \\
S X \\
C Y \\
S Y
\end{array}\right)
$$

\section{Acknowledgement}

I would like to thank Rick Allard for introducing me to LATEX-the special version of TEX used to prepare this document-and for his patience in teaching me how to use it. 


\section{References}

1. G. Guignard, EFFETS DES CHAMPS MAGNETIQUES

PERTURBATEURS D'UN SYNCHROTRON SUR L'ORBITE FERMEE ET LES OSCILLATIONS BETATRONIQUES, AINSI QUE LEUR COMPENSATION, CERN 70-24, 14 Septembre 1970.

2. G. Guignard, "THE GENERAL THEORY OF ALL SUM AND DIFFERENCE RESONANCES IN A THREE-DIMENSIONAL MAGNETIC FIELD IN A SYNCHROTRON', CERN 76-06, 23 March 1976.

3. G. Guignard, 'A GENERAL TREATMENT OF RESONANCES IN ACCELERATORS', CERN 78-11, 10 November 1978.

4. G. K. Green and E. D. Courant, 'THE PROTON SYNCHROTRON', Encyclopedia of Physics, Volume XLIV, pp. 327-328, Springer-Verlag, 1959.

5. E. C. Raka, 'SECOND AND THIRD ORDER STOPBAND CORRECTIONS IN THE AGS', unpublished technical note.

6. C. J. Gardner, 'A REVIEW OF THE LOW-FIELD CORRECTION SYSTEM PRESENTLY EMPLOYED IN THE AGS', AGS/AD/Op. Note No. 17, February 4, 1988.

7. Booster Design Manual.

8. J. Milutinovic, A. G. Ruggiero, S. Tepikian, and W. T. Weng 'AGS-BOOSTER ORBIT AND RESONANCE CORRECTION', Paper presented at The 1989 Particle Accelerator Conference held in Chicago, March 20-23, 1989.

9. S. Tepikian, 'RANDOM SEXTUPOLE CORRECTION', AD Booster Technical Note No. 125, August 5, 1988. 